leuchtenden Teilchen als organische Verunreinigungen von Dr. W o I f f erkannt worden. - Auch das Sublimat zeigt ganz ähnliche Erscheinungen. Hier habe jch zwei Sublimatkonglomerate, von denen clas cine fast gar nicht, das andere intensiv orangerot leuchtet. Chemisch reines Sublimat von $M \in r \mathrm{ck}$ in Darmstadt z. B. zeigt eine Menge gelbrot leuchtender Teilchen. Das reinste $\mathrm{K}$ a $\mathbf{h l}$. b a u m sche Prüparat dagegen zeigt keine Spur von Leuchtrorgang. Ur. Wo If $\mathrm{f}$ hat neuerdings gefunden, daB diese rotleuchtende Substanz Kalomel ist. Hierüber wird Dr. Wo If $\mathrm{f}$ selbst noch näh:res berichten. Auf weitere Substanzen, die ebenfalls fiir die technische ( hemie von Bedeutung sind. will joll mich hiter nicht weiter einlassen. Wie Sie schen, lassen sich nuch der beschrictenen Mlethode auch schon Sipuren von Verunreinigungen recht gut nnchweis:n. Besonders interessant ist, daB die meisten dieser durch louchterscheinungen sich kenntlich machenden Virunreingungen bei den chemisehen Verbindungen nicht in der ganzen Maswe gleichmäBiy verteilt sind, sondern wich in cinigen Krystallen anreichern. Ijese leuchtenden Teilchen nun können so klein gein, dab man sie nit blob:m Auge nicht mehr erkennt, sondern dic lupe oder dis Mikroskop zu Hilfe nehmen muB.

Hicrdurch nun wurde mir der AnlaB gegeben zur Konstruktion des luminescenzmikroskopes, das ich Ihnen leider noch nicht vorführen kann, da es moch nicht vorfïhrungsfähig ist. Dieses Instrument ermöglicht also die mikroskopische Untersuchung von Objekten in ihrem eigenen Fluorescenzlicht, so daB man hiermit auch die letaten Spuren von Lumineseenz beobnchten kann. Ilas Luminescenzmikroskop) dürfte in gleicher Weisc für ('hernik (.r, Mineralogen, Petrographen und Biologen von Bedeutung sein.

In AnschluB an den Fortrag in Freiberg hatte Oberbergrat Prof. I)r. Kolbock die Freundlichkeit, eine Anzahl von .lineralien aus der reichhaltigen Sammlung der Frejberger Bergakndemie dem ultravioletten Licht der CV-Filterlanpe auszusetzen. Fs waren dus folgende lesonders schön leuchtende P'räparate: Fin Diamnnt aus Südafrikn, noch in geinem Mutteryestein, der intensiv blauviolett leuchtete; ferner ein (oelestin aus Sizilien; farbloser FluB. spat aus der tichweiz und violetter aus Cumberland, die beide intensiv blau bis violett leuchten; Wille. mit, nit Kalkspat in einem Stück vorbunden, wobei der Willemit intensiv grün und der Kalkspat rot leuchtete (aus New Jersey U. S. A.); ferner Kalkuranglimmer aus Schwarzenberg im Frzgebirge; Bariunuranglimmer aus Bergen im Vogtlande, der auberordentlich stark grïn leuchtete und, mit dem obengenannten licrnsix.ktroskop) beobachtet, ein scharfe's diskontinuierliches Spektrum ergab; ferner Aragonit aus Böhmen, der weißlich fluorescierte und nach Entfernung aus der Lichtquelle grün phosphorescierte; (diese doppelte Luminescenz ist ein Zeichen, daB an der Luchteracheinung mehrere Substanzen teilnchmen). SchlieBlich Aragonit aus Sizilien, der auf Schwefol gewachsen war. Dieses Mineral war das Glanzatiuck des Vortrages. Es leuchte-te auBerordentlich hellrosenrot und gab ein selir deutlich diskontinuierliches Spektrum. Aus dem ultravioletten Strahlenkegel entfernt, leuchtete (ebenso wie der böhmische Aragonit) intensiv grün nach. Zweifellos werden sich noch an einer ganzen Anzahl Mineralien der reichhaltigen Freiberger Sammlung ebenfalls interessante Leucht erscheinungen feststellen Inssen.

[A. 60.]

\section{Über eine neue Klasse von Gläsern.}

\author{
Von Dr. Ing. Felix Thoyas, Zürich.
}

(Eingeg. 27.4. 1912.)

Erst seit wenigen Munaten erscheint in Handel eine neue Sorte von Gläsern, die das größte Interesse seitens der Glas erzeugenden und verbrauchenden Industrien verdient, vor allem aber für die chemische Grobindustrie von unsclätzharer Bedeutung sein düfte. lch habe bereits in Anfang des Jahres in einem Aufsatz in der ,Chem.-Ztg. " ( Hft. 4, 1912) einige Untersuchungen veröffentlicht, in denen ich auf die wichtigsten Kigenschaften dieser neuen Glasarten hinuies. lie bis jetzt im Handel unter dem Namen ,Siloxydgläser" käuflichen Produkte'hahen physikalisch wie chemisch grobe Ahn. lichkeit mit dem liekannten undurchsichtigen "Quarzglas", von dem sie sich jodoch zunächst dadurch unterycheiden, dab sie eine relativ geringe Menge irgendeines schwerschmelzbaren Uxydes von ausgesprochenem sauren (harakter enthaiten, während day Quarzylas aus völlig reiner, durch Schmelzen in den amorphen Zustand iilergefïlirter Kieselsäure hesteht. Andererseits stehen sie dadurch im (iegensatz zu allen übrigen sog. , (iläsern", da diese durchweg nelien einem mehr oder weniger hohen Kieselsiiuregehalt einen gowiseen und relativ großen Prozentsatz an basischen Metalloxyden enthaiten: letztere sind also löxungen hzw. (iemenge verschiedener silicate (Calcium-Natriumsilicate, Calcium-Aluminiumsilicate, [ev. Aluminate], BleiNatriumsilicate usw.), wobei jedoch ein stöchio. metrisches Verhültnis der einzeloen Komponenten zueinander in der Rexel nicht besteht.

Gläser, die nur aus sauren Oxyden bestehen, waren anscheinend bisher noch nicht bekannt. Wohl kennt unan schon lange einzelne Metalloid-Sauerstoff verbindungen, die Süurecharakter lesitzen und unter Umstïnden die physikalischen kigenschaften des "Glases" im allgemeinen, d. h. um es ctwas joradox auszudrücken: einer "festen Fliissigkeit", in mehr oder weniger deutlichem Maße aufweisen können, wie z. B. die Anlyydride der Bursiiure, arsenigen Süure. Phosplionsiure, Kieselsiiure u. a.

Diese ()xyde können nun aber einzeln als ,Glns“" in technischem Sinne nicht liezcichnet und verwendet werden, mit nlleiniger Ausnahme des geschmolzenen Siliciumdioxydes. Wohl dienen Borsäure-, Arsenigsïure-, Phusphoräureanhydrid als Zusätze bei der Glasfabrikation, aber nur in geringen Mengen, da sie unverhunden leichl wasser-, alkali-oder süurelöslich sind, und meist auch ihr amorpher Kustand nicht sehr stabil ist. Letztere Erscheinung zeigt -- allerdings erst hei hohen Temperaturen - auch das cinzige dieser Oxyde," das, wenn amorph, für sich allein ein vollkommenes Glas in physikalischem Sinne durstellt, nämlich das Sili- 
ciumdioxyd, das in diesem Falle, wie hekannt, als Quarzglas bezeichnet wird.

Es dürfte in Fachkreisen hinreichend bekannt sein, daß dieses Verhalten, das sog. „Entglasen“, des für viele $/ w$ recke wegen seiner besonderen thermischen und chemischen Eigenschaften fast unentbehrlich gewordenen Quarzglases beim Arbeiten in höhsren Temperaturen (oberhall, $1200^{\circ}$ ) stets sehr atörend zu wirken pflegt; in kurzer Zeit werden duroh die Entglasung, d. h. durch den tbergang aus dem amorphen in den krystallinen Zustand, die benutzten ('eriite (P'yrometerröhrchen. Tiegel, Heizrohre, Muffeln. Verbrennungwschiffehen usw.) völliz unbrawhhar. weil infolgo dieser Lmwandlung zunüchst eine Volumverminderung und Gifïgeänderung stattfindet, wodureh bewirkt wird, daB das Material seine mechanische festigkeit und einen reiner wichtigsten und bekanntesten Vorzïgo verliert: die Linemptindlichkeit gegen schroffen Temperaturwechsel.

Fis gilt dies vor allem ron dem technisehen Quarzqlas. das im Gegensatz zu dem fïr Laboratoriumsgerïte verwendeten, undurchsichtig lis durchscheinend ist. Bekanntlich hat nun die chemische (irobindustric an ersterem ein bedeutendes Interesise, einmal, woil es eine hervorragende Säurefentigkeit und 'Temperaturhostïndigkeit besitzt, dann aher auch, weil es relativ wohlfeil ist, während das durchsichtige Mnterial zurzeit wegen seines holien Preises nur beschrïnkte Verwendung finden kann.

lch hale mich aus diesen (irunde der intereswanten Aufgabe unterzogen, festzustellen, ol, und durch welehe llittel sich das technische Quarzglas verhessern, baw. sicll eine neue (ilassorte finden ließe, die wohl dic Vorzüge des ersteren aufweist, alier soweit wie möglich von dessen Mängeln frei ist. Dic Anregung zu diesen Untersuchungen verdanke ich Herrn P'rof. W. B o r c he rs, Aachen, welcher in (iemeinschaft mit Dr. F. WolfB u rekhar d t. Biebrich a. Rh., gefunden hatte, dab durch 'Zuxïty' von sauren (Ixyden zur Kieselsïuro eine neue Art (i]ïser hergestelit werden kann, insbesondere. dals die technischen Eigenschaften des Quarzylases durch Zusatz, selbst geringer Mengen von Zirkonoxvil oder Titansäureanhydrid zu demsellsen ganz crlieblich beeinfluBt wurden.
In meiner oben erwähnten Veröffentlichung in der Chem.-Ztg hatte ich nun die Ergebnisse meiner ersten Untersuchungen in dieser Richtung zusammengestellt, wobei zunächst der Einflu $B$ von Zirkonoxyd und Titansäureanhydrid festgestellt worden war. Das Ergebnis dieser Untorsuchungen war in Anbetracht der relativ selhr geringen Mengen der zugesetzten Oxyde ein überraschendes: Reine Kieselsäure, mit den erwähnten Oxyden schon in Mengen von $0,1 \%$ an verschmolzen, tiefert ein (ilas (nach dem zugesetzten Oxyd "Zirkonglas" hzw. "Titanglas" benannt), das naturgemäß reinem Quarzglas sehr ähnlich ist; es besitzt dieselbe ln. empfindlichkeit gegen schroffen Temperaturwechsel, dieselbe sïurefestigkeit, mindestens den gleich hohen schmelzpunkt, und ist ein ebenso guter Is(). lator wie Quarzglas. Dagegen halien die ., Siloxyd. gläser" wie diese neue Glassorte benannt wurde. eine nicht unbedeutende höhere $\left(20-50^{\circ}{ }_{(1)}\right)$ mecha nische Festigkeit, grö̈lsere Härte und geringere Sprödigkeit; ferner sind sie bedentend widerstandsfähiger gegen hasische Metalloxyde (mit Ausnalıme der eigentlichen Alkalien), aucl, bei lochen Temperaturen.

Jer wichtigste Vorteil gegeniiher Qunrzglas ist der, daß die Entglasung der Siloxydyläser bei weitem nicht so leicht eintritt wio die des ersteren; eine Tatsache, deren Ursachen ich bisher noch nicht völlig habe aufklären können. In meiner erwiihnten Veröffentlichung iuljer diesen ('egenstand habe ir.h - auf Grund der chemischen Analyse und des mikroskopischen Befundes -- die Vermutung allsgesprochen. daB es sich um eine lösung von sauren Oxyden oder deren Silicaten in überschüssiger Kieselsiuure handele. Ich hin zur Zeit noch mit der Untersuchung mir äberlaswener Glïser hesehäftigt. besonders aber mit einem, das anscheinend nuch weit geringeres Entglasungsbestreben zeigt als alle bisher untersuchten Siloxydgläser. Ich glaube annehmen zu diirfen, dab wir es hier mit kolloidalen Lösungen von sauren Oxyden in Kieselsiiureanhydrid zu tun haben und diese Annahme würde auch in gewissem Sinne eine Erklürung für die autfïllige Verminderung der Entglasungstendenz zulassen. lch erwarte von der fortsetzung meiner Untersuchungen eine Klürung dieser Frage und hoffe, bald hierüber und über die neuesten siloxydgläser berichten zu können.

[A. 98.

\section{Wirtschaftlich-gewerblicher Teil.}

Dic Berliner chemlsehen und verwandten Industrien sind ron dem allgemeinen Aufschwung, den Industrie und Gowerbe genommen haben, ebenfalls beeinflubt worden, wenn dies auch nicht auf sämtliche Zweige zutreffen kinn. Was die anorganische chemische Industric anbetriff, ist es nur bei einem kleinen Teil der Produkte gelungen, geringe Preiserhöhungen zu erzielen, im allgemeinen ist jedoch der Prois vieler Artikel, liesonders der Ammoniakproduktion, mit den höheren Preisen für Roh-und Verbrauchsnaterialien nicht in Hinklang zu bringen.

Der Absatz von S c h w e f e I s ä u ro, sowohl konzentriert als alu'h in den schwächeren Sorten, ist ziemlich befriedigend. Die Preise konnten hier und da otwas aufgebessert werden. Salzsäure konnte nicht immer in genïgenden Mengen angeboten werden. Die Preise sind bei der regen Nachfrage et way höher. Die Proise für Salpetersäure konnten nicht immer den Kosten des Chilesalpeters angepaBt werden. Der Absatz ist einigermaßen hefriedigend. Für calciniertes Glaubersalz entwickelt sich eine merklich steigende Nachfrage, so daB die eingehenden Aufträge nicht immer rochtzeitig aungeführt werden könnon. Die wenig gewinnbringenden $V(r .5$ kaufspreise konnten in einzelnen Fällen otwas erhöht werden. Salmiakgeist war sehr begehrt. Die Preise konnten fast ïberall erhöht werden, duch steben sie noch nicht im richtigen Verhältnis zu 